\title{
Alterações físico-químicas de geleias de melão e acerola durante o armazenamento
}

\author{
Physical and chemical characteristics of acerola and melon jellies during storage \\ Cristiane Alves de Paiva ${ }^{1}$ Edna Maria Mendes Aroucha ${ }^{2}$, Rafaella Martins de Araújo Ferreira ${ }^{3}$, Nícolas Oliveira de Araújo ${ }^{4}$, \\ Paulo Sérgio Lima e Silva
}

\begin{abstract}
Resumo: O processamento de polpa e a fabricação de geleias são produtos de interesse para a região nordeste do Brasil. A baixa precipitação e os altos índices de luminosidade anuais, associado ao desenvolvimento da irrigação localizada na região, atraíram grandes empresas nacionais e internacionais para a produção de variados tipos de frutos, incluindo a acerola e o melão. O objetivo do presente trabalho foi avaliar as características físico-químicas de geleia simples e mista (50\% de melão e $50 \%$ de acerola) de melão e acerola, por ocasião do preparo e após o armazenamento por 30 dias. Utilizou-se o delineamento inteiramente casualizado. O tempo de armazenamento aumentou o teor de sólidos solúveis (SS) e o pH, mas reduziu a acidez titulável e o teor de ácido ascórbico das geleias. A geleia de melão apresentou o maior teor de SS após o armazenamento. E a geleia de acerola obteve valores superiores de $\mathrm{pH}$, acidez titulável e vitamina $\mathrm{C}$, nas duas épocas de análise. Já a geleia mista apresentou pH igual à geleia de melão, mas quanto às demais características situou-se entre às geleias simples.
\end{abstract}

Palavras-chave: ácido ascórbico; Cucumis melo; geléia mista; Malpighia emarginata; sólidos solúveis

\begin{abstract}
The pulp processing and the manufacture of jellies are products of interest to the northeastern region of Brazil. The low rainfall and high rates of annual luminosity associated with the development of drip irrigation in the region, attracted large national and international companies for the production of various kinds of fruits, including acerola and melon. The objective of this study was to evaluate the physico-chemical characteristics of single and mixed jelly (50\% of melon and 50\% acerola) of melon and acerola, on the occasion of preparation and after storage for 30 days. We used a completely randomized design. The storage time increased the soluble solids (SS) and $\mathrm{pH}$, but reduced titratable acidity and ascorbic acid content from jelly. The melon jelly had the highest SS content after storage. And acerola jelly obtained higher values of $\mathrm{pH}$, titratable acidity and vitamin $\mathrm{C}$, in two periods of analysis. However the mixed jelly showed $\mathrm{pH}$ seems to jelly melon, but to the other features it was between the simple jelly.
\end{abstract}

Keywords: ascorbic acid; Cucumis melo; Malpighia emarginata; mixed jelly; soluble solids;

\footnotetext{
*Autor para correspondência

Recebido para publicação em 08/05/2015; aprovado em 13/07/2015

${ }^{1}$ Engenheira Agrônoma, Mestranda em Fitotecnia, Universidade Federal Rural do Semiárido (UFERSA), Mossoró /RN. E-mail: cristiane_uzl@ hotmail.com

${ }^{2}$ Dra. em Produção Vegetal, Profa. Adjunto IV da Universidade Federal Rural do Semiárido (UFERSA), Mossoró/RN. E-mail: aroucha@ufersa.edu.br

${ }^{3}$ Engenheira Agrônoma, Doutoranda em Fitotecnia, Universidade Federal Rural do Semiárido (UFERSA), Mossoró/ RN. E-mail: rafaellamarafe@ gmail.com

${ }^{4}$ Graduando em Agronomia, Universidade Federal Rural do Semiárido (UFERSA), Mossoró/RN. E-mail: nicolas_araujo1892@hotmail.com

${ }^{5}$ Dro. em Agronomia (Genética e Melhoramento de Plantas) Professor do Departamento de Ciências Vegetais da Universidade Federal Rural do Semiárido (UFERSA). Mossoró - RN. E-mail: paulosergio@ufersa.edu.br
} 


\section{INTRODUÇÃO}

Geleia é o produto obtido pela cocção de frutas inteiras ou em pedaços e, ou, sucos ou extratos aquosos de frutas, misturados com açúcares, com ou sem adição de água, pectina, ácidos e outros ingredientes, até a obtenção de uma consistência semissólida adequada, convenientemente processada seja obtida, e adequadamente condicionada de forma a assegurar sua perfeita conservação (BRASIL, 1978).

As geleias podem ser elaboradas com uma ou mais espécies de frutas sendo, portanto, designadas como simples ou mistas, respectivamente. O produto pode ser do tipo comum ou extra, segundo a proporção de suco e fruta utilizada. A primeira é elaborada com 40 partes do fruto e 60 partes de açúcar, enquanto na segunda a proporção é de $50 \%$ de frutas e o restante de açúcar (BRASIL, 1978).

$\mathrm{Na}$ preparação de geleia a legislação brasileira estabelece um pH máximo de 3,4, acidez titulável de no mínimo $0,3 \%$ e no máximo $0,8 \%$ e um teor mínimo de $65^{\circ}$ Brix de sólidos solúveis (JACKIX, 1988).

Existem várias razões para a produção de geleias no Brasil. As frutas tropicais são altamente perecíveis, deteriorando-se em poucos dias, e a fabricação de geleias reduz a perda dessas frutas (MÉLO et al., 1999). Algumas frutas possuem a produção concentrada em poucos meses e seriam perdidas, caso não fossem utilizadas alternativamente como, por exemplo, na produção de geleias (Mota 2006). Subprodutos da industrialização de algumas frutas podem ser utilizados na fabricação de geleias (SILVA et al., 2012). A fabricação de geleias permite a utilização de frutas pouco conhecidas ou muito ácidas in natura (SOUZA FILHO et al., 2002). Finalmente, a fabricação de geleias agrega valor às frutas e possibilita a produção de novos sabores (FERREIRA et al., 2010).

A cultura do meloeiro, por exemplo, possui uma relevante importância para a fruticultura, sobretudo na região semiárida brasileira (FREITAS et al., 2014), (Agrianual., 2009). Seu fruto tem elevado $\mathrm{pH}$, baixa acidez titulável $(0,14 \%)$ e sólidos solúveis acima de 9\% (TOMAZ et al., 2009). Na agroindústria do processamento mínimo de melão cerca de $60 \%$ da matéria-prima é descartada como resíduo, dessa forma a fabricação de doces e geleias são alternativas que possibilitam a agregação de valor (Miguel et al., 2008). Em estudo realizado com a finalidade de estudar alternativas para o aproveitamento das cascas e das sobras de polpa de melões minimamente processados, (MIGUEL et al.,2008) observaram que a geleia do respectivo fruto obteve uma boa aceitabilidade por parte dos provadores.

A acerola (Malpighia emarginata DC.) é outro fruto que tem ganhado destaque em termos de produção no Nordeste (BASF, 2006). Em virtude dos seus atributos de qualidade como vitamina $\mathrm{C}$ e carotenoides, com teores de $347,52 \mu \mathrm{g} .100 \mathrm{~g}^{-1}$ e $1699,01 \mathrm{mg}$ de ácido ascórbico. $100 \mathrm{~g}^{-1}$ respectivamente (AQUINO et al.,2011), este fruto apresenta potencial para industrialização e dessa forma tem sido objeto de estudo para a produção de geleias (MÉLO et al.,1999), (MATTA et al., 2004), (MACIEL et al.,2009), (Sousa et al., 2010), (CAETANO et al., 2012).

O período de conservação, das características físicoquímicas, sensoriais e microbiológica, de um produto processado é importante para que o consumidor obtenha um produto com qualidade. Porém, sabe-se que o prazo de validade do produto pode variar conforme as condições de embalagem e temperatura de conservação. (CARDOSO et al., 1997) observaram decréscimo da cor vermelha ao longo do tempo de armazenamento da geleia de jambo, com influência direta da luz e temperatura.

Apesar de estar disponível na literatura alguns trabalhos de armazenamento de geleias de frutas, a exemplo de umbu-cajá (OLIVEIRA et al., 2014), goiaba (MESQUITA et al., 2013) e entre outros, ainda não existem estudos sobre a avaliação das alterações físico-químicas de melão e acerola durante o armazenamento. Nesse contexto, o objetivo do presente trabalho foi avaliar as características físico-químicas de geleia simples e mista (50\% de melão e $50 \%$ de acerola) de melão e acerola, por ocasião do preparo e após o armazenamento por 30 dias.

\section{MATERIAL E MÉTODOS}

Os frutos (acerola e melão) usados nas formulações das geleias foram obtidos no pomar do assentamento MAISA e em seguida levados para o Laboratório de Pós-colheita do DACS, onde procedeu-se o preparo das mesmas. Para ser utilizada na formulação das geleias, as acerolas, previamente lavadas, foram prensadas, sendo a polpa separada do suco com o auxílio de uma peneira. $\mathrm{O}$ melão foi seccionado para a retirada da polpa e esta foi homogeneizada com auxílio de um mixer.

As geleias foram elaboradas em três formulações: $100 \%$ acerola, $50 \%$ acerola: $50 \%$ melão e $100 \%$ melão. Cada tipo de geleia foi elaborada utilizando-se uma proporção de polpa: açúcar de 1:0,5. Procedeu-se à cocção em panela de aço inoxidável com capacidade para dois litros, com agitação manual contínua até concentração final de sólidos solúveis de $68 \%$, medido em refratômetro. Tal concentração foi adotada também por (NACHTIGALL et al., 2004). Após esta etapa, a geleia foi envasada à quente em embalagens de vidro com capacidade para $250 \mathrm{~g}$, previamente esterilizadas a $121^{\circ} \mathrm{C} / 15$ min; fechadas com tampa de metal; imediatamente resfriadas por adição de água fria por 15 minutos. As geleias foram mantidas à temperatura ambiente $\left(23{ }^{\circ} \mathrm{C}\right.$ e $60 \%$ UR $)$ e avaliadas em intervalos de armazenamento de 0 e 30 dias, de acordo com parâmetros visuais e físico-químicos.

$\mathrm{O}$ experimento foi conduzido em delineamento inteiramente casualizado, com esquema fatorial $3 \times 2$ (três formulações de geleia e dois períodos de armazenamento) e três repetições. As seguintes análises físico-químicas foram realizadas, em triplicata, conforme metodologia específica: $\mathrm{pH}$ - determinado utilizando potenciômetro digital; sólidos solúveis (SS) - utilizando o refratômetro modelo SAMMAR 852610472 e os resultados foram expressos em porcentagem (\%); acidez titulável (AT) - foi determinada através da titulação com solução de $\mathrm{NaOH}(0,1 \mathrm{~N})$, previamente padronizada, os resultados expressos em mEq/100g; e vitamina $\mathrm{C}$ (VitC) - foi obtida por titulação com DFI $(2,6$ diclorofenol-indofenol 0,02\%) e expresso em $\mathrm{mg}$ de ácido ascórbico por $100 \mathrm{~g}$ de geleia.

Os dados das variáveis estudadas foram submetidos à análise de variância através do programa estatística SISVAR (FERREIRA., 2003) e suas médias comparadas pelo teste de Tukey ao nível de 5\% de probabilidade. 


\section{RESULTADOS E DISCUSSÃO}

Verificou-se interação significativa dos fatores tipos de geleias e tempo de armazenamento em todas as características avaliadas (Tabela 1 e 2). Os aumentos (no teor de sólidos solúveis e no $\mathrm{pH}$ ) e as diminuições (na acidez titulável e no teor de ácido ascórbico) verificados no presente trabalho (Tabelas 1 e 2) foram diferentes nos tipos de geleias, em resposta ao período de armazenamento, o que explica o efeito da interação tipos de geleias $\mathrm{x}$ tempos de armazenamento (Tabelas 1 e 2). Resultados semelhantes a estes foram observados na avaliação de geleias simples e mistas de melancia (Citrullus vulgaris S.) e tamarindo (Tamarindus indica L.) (FERREIRA et al., 2010).

Não houve diferença entre geleias, quanto ao teor de sólidos solúveis (SS), na avaliação feita imediatamente após o preparo das geleias (Tabela 1). Porém, após 30 dias de armazenamento, a geleia de melão apresentou o maior SS e a geleia de acerola, o menor. A mistura dos dois tipos de geleias apresentou SS intermediário (Tabela 1). O teor de SS aumentou nos três tipos de geleias testados, após 30 dias de armazenamento (Tabela 1). Outros autores (Selvamuthukumaran et al.,2007), ( SAFDAR et al., 2012), (ASSIS et al., 2007), (MUHAMMAD et al., 2008) também verificaram aumento teor no de SS, com o armazenamento das geleias. Entretanto, (ARÉVALO-PINEDO et al.,2013) observaram que o teor de SS permaneceu constante com o armazenamento nas geleias de araticum (Araticum Crassiflora Mart.)

A acidez dos três tipos de geleias, medida pelo $\mathrm{pH}$, aumentou com o aumento no período de armazenamento (Tabela 1). Nas duas avaliações feitas no presente trabalho, a geleia de acerola foi menos ácida do que os outros tipos de geleias (Tabela 1). Os resultados dos efeitos do período de armazenamento sobre o $\mathrm{pH}$ das geleias são conflitantes. Fato semelhante ao constatado no presente trabalho foi observado com a geleia de Fragaria ananassa (ISLAM et al., 2012b) e de abacaxi (LICODIEDOFF et al., 2010). (FERREIRA et al., 2010), por outro lado, verificaram que, na geleia mista melancia-tamarindo, o $\mathrm{pH}$ aumentou 30 dias após o armazenamento (DAA), mas diminuiu 60 DAA. (ARÉVALO-PINEDO et al.,2013) verificaram que, na geléia "convencional" de araticum (Annona crassiflora Mart), o pH aumentou e depois diminuiu, com o aumento do tempo de armazenamento. Entretanto, na geleia light (substituição da sacarose pela sucralose), o pH permaneceu constante por até seis meses, o que também ocorreu na geléia de caju (Anacardium occidentale L.) por quatro meses (Assis et al., 2007). Enquanto, (ISLAM et al.,2012a) verificaram que o $\mathrm{pH}$ diminuiu com o tempo de armazenamento da geleia de Hylocereus undatus (pitaia).

Tabela 1. Características de geleias armazenadas sob condição ambiente (temperatura e umidade relativa do ar de $23{ }^{\circ} \mathrm{C}$ e $70 \%$, respectivamente) $)^{1}$

\begin{tabular}{lcccc}
\hline \multirow{2}{*}{ Geleia } & \multicolumn{2}{c}{ Sólidos solúveis (\%) } & \multicolumn{2}{c}{$\mathrm{pH}$} \\
\cline { 2 - 5 } & \multicolumn{2}{c}{ Tempo de armazenamento (dias) } & \multicolumn{2}{c}{ Tempo de armazenamento (dias) } \\
\cline { 2 - 5 } & 0 & 30 & 0 & 30 \\
\hline Acerola & $69,0 \mathrm{aC}$ & $71,0 \mathrm{cA}$ & $3,37 \mathrm{aB}$ & $3,64 \mathrm{aA}$ \\
\hline $50 \%$ acerola $+50 \%$ melão & $69,3 \mathrm{aB}$ & $71,8 \mathrm{bA}$ & $3,25 \mathrm{bB}$ & $3,50 \mathrm{bA}$ \\
\hline Melão & $69,0 \mathrm{aB}$ & $73,0 \mathrm{aA}$ & $3,07 \mathrm{cB}$ & $3,45 \mathrm{bA}$ \\
\hline
\end{tabular}

${ }^{1}$ Médias seguidas pela mesma letra minúscula, nas colunas, e pela mesma letra maiúscula, nas linhas, não diferem entre si, a 5\% de probabilidade, pelo teste de Tukey.

Nas duas avaliações, as maiores médias da acidez titulável (AT) foram apresentadas pela geleia de acerola e as menores pela geleia de melão. A mistura da geleia dos dois tipos de frutos apresentou AT intermediária (Tabela 2). A AT diminuiu com o aumento do período de armazenamento, nos três tipos de geleias (Tabela 2).(LEÃO et al.,2012) também verificaram reduções na AT com os aumentos nos períodos de armazenamento na geleia de mamão. Na polpa da goiaba e no suco da acerola também foram observadas reduções na acidez titulável com o aumento do período de armazenamneto (FREITAS et al., 2006), ( BRUNINI et al., 2003). Aumentos nesta característica, com o aumento do tempo de armazenamento foram observados por (SELVAMUTHUKUMARAN et al., 2007). Mas outros autores verificaram, em geleia de caju (Anacardium occidentale L.), que a AT permaneceu constante por até 120 dias após o preparo (ASSIS et al., 2007). Constância semelhante foi observada por (ARÉVALO-PINEDO et al.,2013), em geleia de araticum, mas um ligeiro decréscimo foi constatado antes da última amostragem, realizada antes dos 180 dias.

ORDÓNEZ-SANTOS e VÁSQUEZ-RIASCOS ,2010) constataram "alterações erráticas" no pH e na acidez titulável, em função do tempo de armazenamento, e, segundo eles, isso foi observado também por outros autores. Essas alterações consideradas erráticas, que foram observadas também no teor de ácido ascórbico talvez estejam associadas ao tipo de análise empregado por vários autores no estudo de características físico-químicas de frutos em função do armazenamento. Muitos autores aplicam testes de médias quando, na realidade, o correto seria a aplicação de uma análise de regressão. 
Tabela 2. Características de geleias armazenadas sob condição ambiente (temperatura e umidade relativa do ar de $23{ }^{\circ} \mathrm{C}$ e $70 \%$, respectivamente $)^{1}$

\begin{tabular}{|c|c|c|c|c|}
\hline \multirow[t]{3}{*}{ Geleia } & \multicolumn{2}{|c|}{ Acidez titulável (mEq/100 g) } & \multicolumn{2}{|c|}{ Ácido ascórbico $(\mathrm{mg} / 100 \mathrm{~g})$} \\
\hline & \multicolumn{2}{|c|}{ Tempo de armazenamento (dias) } & \multicolumn{2}{|c|}{ Tempo de armazenamento (dias) } \\
\hline & 0 & 30 & 0 & 30 \\
\hline Acerola & $17,55 \mathrm{aA}$ & $9,19 \mathrm{aB}$ & $2.657,5 \mathrm{aA}$ & $241,1 \mathrm{aB}$ \\
\hline $50 \%$ acerola $+50 \%$ melão & $16,13 \mathrm{bA}$ & $8,33 \mathrm{bB}$ & $1.828,0 \mathrm{bA}$ & $114,5 \mathrm{bB}$ \\
\hline Melão & $15,27 \mathrm{cA}$ & $7,69 \mathrm{cB}$ & $1.143,7 \mathrm{cA}$ & $58,5 \mathrm{cB}$ \\
\hline
\end{tabular}

Nas duas avaliações, o teor de ácido ascórbico (AA) foi maior na acerola, menor no melão e intermediário na geleia mista (Tabela 2). O teor de AA diminuiu com o armazenamento, nos três tipos de geleias (Tabela 2). Outros autores (ASSIS et al., 2007), (MUHAMMAD et al., 2011), (ARÉVALO-PINEDO et al., 2013), à semelhança do presente trabalho, verificaram redução no teor de AA, no decorrer do tempo de armazenamento, em vários tipos de geleias.

O aumento no teor de sólidos solúveis durante o armazenamento verificado neste trabalho pode ser devido à hidrólise ácida de polissacarídeos, especialmente amido e pectina (SAFDAR et al., 2012) (Tabela 1). Alguns autores atribuíram o decréscimo no $\mathrm{pH}$ de algumas geleias, tal como observado no presente trabalho (Tabela 1), à formação de ácidos livres e à hidrolise de pectina (IMRAN et al., 2000). (ORDÓNEZ-SANTOS e VÁSQUEZ-RIASCOS, 2010) acreditam que as mudanças no $\mathrm{pH}$ e na acidez titulável podem ter sido devidas aos efeitos combinados de lixiviação e oxidação de ácidos orgânicos na matriz biológica (Tabela 2). A identificação de fatores responsáveis pela redução dos teores de ácido ascórbico, em resposta ao armazenamento, observada no presente trabalho, fica dificultada porque a diminuição depende de uma série de fatores incluindo temperatura, $\mathrm{pH}$, incidência de luz, presença de oxigênio e de compostos metálicos (GABAS et al., 2003), (SILVA et al., 2006), (OLIVEIRA et al., 2010). Não obstante, vale mencionar que o ácido ascórbico é um antioxidante natural e sua tendência, em um alimento, é oxidar-se, tendo degradação acelerada com o tempo de armazenamento (LIMA et al., 2007).

$\mathrm{Na}$ preparação de geleias, a legislação brasileira estabelece um pH máximo de 3,4, acidez titulável de, no mínimo $0,3 \%$ e de no máximo $0,8 \%$, e um teor mínimo de 65 $\%$ de sólidos solúveis totais (JACKIX, 1988). O teor de sólidos solúveis ideal para geleias é de 67,5. Para valores menores de 64, o gel torna-se fraco e acima de 71 ocorre a cristalização da geleia (JACKIX, 1988). Portanto, as geleias que serviram de base ao presente trabalho (Tabelas 1 e 2) apresentam valores de características físico-químicas incluídos nos limites estabelecidos pela legislação nacional.

Conforme ressaltado, existem discordâncias entre estudos quando aos efeitos do armazenamento sobre o teor de sólidos solúveis totais, $\mathrm{pH}$ e acidez titulável das geleias. Em parte estas discordâncias devem-se a características das frutas empregadas na elaboração das geleias. Apoiando esta proposição está o fato de frutos ou geleias de diferentes genótipos da mesma cultura diferirem quanto a atributos físico-químicos (MATSUURA et al., 2001; SAFDAR et al., 2012). De acordo com (ALBUQUERQUE ,1997), o pH da geleia é resultado da acidez característica da fruta empregada e pode apresentar variações durante o armazenamento como demonstrado por (NACHTIGALL et al., 2004) com geleia de amora elaborada com $0,5 \%$ de pectina cujo $\mathrm{pH}$ inicial foi de 3,10 e após 90 dias decresceu para 3,00. Outros fatores que contribuem para explicar as discordâncias referidas incluem o modo de preparo das geleias, o período de avaliação e até mesmo a maneira de avaliar estatisticamente os resultados. Aparentemente, as menores discordâncias entre estudos referem-se ao teor de ácido ascórbico (AA) das geleias. Isto deve acontecer porque vários fatores contribuem para a degradação desta característica, de modo que o período de armazenamento tende, em geral a reduzir o teor de AA.

\section{CONCLUSÕES}

1. O tempo de armazenamento aumentou o teor de sólidos solúveis (SS) e o $\mathrm{pH}$, mas reduziu a acidez titulável e o teor de ácido ascórbico das geleias;

2. A geleia de melão apresentou o maior teor de SS após o armazenamento. E a geleia de acerola obteve valores superiores de $\mathrm{pH}$, acidez titulável e vitamina $\mathrm{C}$, nas duas épocas de análise. Já a geleia mista apresentou $\mathrm{pH}$ igual à geleia de melão, mas quanto às demais características situouse entre às geleias simples.

\section{REFERÊNCIAS BIBLIOGRÁFICAS}

AGRIANUAL: Anuário estatístico da agricultura brasileira. São Paulo: FNP Consultoria \& Comércio, 2009.

ALBUQUERQUE, J. P. Fatores que influenciam no processamento de geleias e geleiadas de frutas. Boletim da Sociedade Brasileira de Ciência e Tecnologia de Alimentos, v. 15, n. 3, p. 268-278, 1997.

AQUINO, A.C.M.S.; MÉS, R.S.; CASTRO, A. A. Estabilidade de ácido ascórbico, carotenoides e antocianinas de frutos de acerola congelados por métodos criogênicos. Brazilian Journal of Food Technology, v.14, n.2, p. 154-163, 2011.

ARÉVALO-PINEDO, A.; CARNEIRO, B. L. A.; ZUNIGA, A. D. G.; ARÉVALO, Z. D. S.; SANTANA, A. A.; PINEDO, R. A. Alterações físico-químicas e colorimétricas de geléias de araticum (Annona crassiflora). Revista Brasileira de Produtos Agroindustriais, v.15, n.4, p.397-403, 2013.

ASSIS, M. M. M.; MAIA, G. A.; FIGUEREDO, E. A. T.; FIGUEREDO, R. W.; MONTEIRO, J. C. S. Processamento e estabilidade de geleia de caju. Revista Ciência Agronômica, v.38, n.1, p.46-51, 2007. 
BRASIL. Diário Oficial da República Federativa do Brasil. Resolução normativa n.15, 4 de maio de 1978. Regulamento técnico para fabricação de geleia de frutas. Brasília, 1978.

BRUNINI, M.A.; OLIVEIRA, A.L.; VARANDA, D.B. Avaliação da qualidade de polpa de goiaba 'Paluma' armazenada a $-20{ }^{0} \mathrm{C}$. Revista Brasileira de Fruticultura, v.25, n.3, p.394-396, 2003.

CAETANO, P.K.; DAIUTO, E.R.; VIEITES, R.G. Característica físico-química e sensorial de geleia elaborada com poula e suco de acerola. Brazilian Journal of Food Technology, v.15, n.3, p.191-197, 2012.

CARDOSO, R. L.; FERREIRA, V. L. P.; MONTGOMERY, M. W.; YOTSUYANAGI, K. Efeito do tempo, luz e temperatura na cor da geléia de jambo vermelho (Eugeniamalaccensis, Lin). Ciência e Tecnologia de Alimentos, v. 17, p. 28-31, 1997.

BASF S. A. Unidades de Produtos para Fruticultura. Frutas para exportação. Atualidades agrícolas: fruticultura o sucesso do Vale São Francisco. São Bernardo do Campo, n. 6, p.16-29, 2006.

Ferreira, D. F. Programa de análises estatísticas (statistical analysis sotware) e planejamento de experimentos SISVAR 5.0 (Build 67). Lavras: DEX/UFLA, 2003.

FERREIRA, R.M.A.; AROUCHA, E.M.M.; SOUSA, A.E.D.; MELO, D.R.M.; PONTES FILHO, F.S.T. Processamento e conservação de geleia mista de melancia e tamarindo. Revista Verde, v.5, n.3, p.59-62, 2010 .

FREITAS, C.A.S.; MAIA, G.A.; COSTA, J.M.C.; FIGUEIREDO, R.W.; RODRIGUES, M.C.P.; SOUSA, P.H.M. Estabilidade do suco tropical de acerola (Malpighia emarginata D.C.) aadoçado envasado pelos processos hot-fill e asséptico. Ciência e Tecnologia de Alimentos, v.26, n.3, p.544-549, 2006.

FREITAS, L. D. A.; FIGUEIRÊDO, V. B.; PORTO FILHO, F. Q.; COSTA, J. C.; CUNHA, E. M. Crescimento e produção do meloeiro cultivado sob diferentes níveis de salinidade e nitrogênio. Revista Brasileira de Engenharia Agrícola e Ambiental, v.18, (Suplemento), p.20-26, 2014.

GABAS, A.L.; TELIS-ROMERO, J.; MENEGALLI, F.C. Cinética de degradação do ácido ascórbico em ameixas liofilizadas. Ciência e Tecnologia Alimentar, v.23, p.66-70, 2003.

IMRAN, A.; KHAN, R.; AYUB, M. Effect of added sugar at various concentration on the storage stability of guava pulp. Sarhad Journal of Agriculture, v.16, n.1, p.8993, 2000.

ISLAM, M.Z.; KHAN, M.T.H.; HOQUE, M.M.; RAHMAN, M.M. Studies on the processing and preservation of dragon fruit (Hylocereus undatus) jelly. The Agriculturist, v. 10, n.2, p.29-35, 2012a.

ISLAM, M.Z.; MONALISA, K.; HOQUE, M.M. Effect of pectin on the processing and preservation of strawberry (Fragaria ananassa) jam and jelly. International Journal of Natural Sciences, v.2, n.1, p.8-14, 2012b.

JACKIX, M. H. Doces, geléias e frutas em calda: Teórico e Pratico. Campinas: Ed. Icone, 1988.

LEÃO, K.M.M.; BOUDOU, F.S.T.; CASTRO, A.A.; FIGUEIREDO, A.V.D. Formulação e avaliação físicoquímica de geleia de mamão (Carica papaya L.). Scientia Plena, v.8, n.3, p.1-3, 2012.

LICODIEDOFF, S.; GODOY, R.C.B.; AQUINO, A.D.; VIANA, A.D. Geleia de Abacaxi: influência do tipo de pectina nas alterações físico-químicas durante o armazenamento. Embrapa, 2010. 9p. (Comunicado Técnico 134).

LIMA, E. S.; SILVA, E. G.; MOITA NETO, J.M.; MOITA, G. C. Redução de vitamina $\mathrm{C}$ em suco de caju (Anacardium ocidentale L.) industrializado e cajuína. Quimica Nova, v.30 n.5, p. 1143-1146, 2007

MACIEL, M.I.S.; MELO, E.A.; LIMA, V.L.A.G.; SILVA W.S.; MARANHÃO, C.M.C.; SOUZA, K.A. Características sensoriais e físico-químicas de geleias mistas de manga e acerola. Boletim do Centro de Pesquisa de Processamento de Alimentos, v.27, n.2, p.247-256, 2009.

MATSUURA, F. C. A. U.; CARDOSO, R. L.; FOLEGATTI, M. I. S.; OLIVEIRA, J. R. P.; SANTOS, D. B. Avaliações físico-químicas em frutos de diferentes genótipos de acerola (Malpighia punicifolia L.) Revista Brasileira de Fruticultura, v. 23, n. 3, p. 602-606, 2001.

MATTA, V.M.; CABRAL, L.M.C.; SILVA, L.F.M. Suco de acerola microfiltrado: avaliação da vida-de-prateleira. Ciência e Tecnologia Alimentar, v.24, n.2, p.293-297, 2004.

MÉLO, E.A.; LIMA, V.L.A.G.; NASCIMENTO, P.P. Formulação e avaliação físico-química e sensorial de geleia mista de pitanga (Eugenia uniflora L.) e acerola (Malpighia sp). Boletim do Centro de Pesquisa de Processamento de Alimentos, v.17, n.1, p.33-44, 1999.

MESQUITA, K. S.; BORGES, S. V.; CARNEIRO, J. D. S.; MENEZES, C. C.; MARQUES, G. R. Quality alterations during storage of sugar-free guava jam with added prebiotics. Journal of Food Processing and Preservation, v.37, p.806-813, 2013.

MIGUEL, A. C. A.; ALBERTINI, S.; BEGIATO, G. F.; DIAS, J. R. P. S.; SPOTO, M. H. F. Aproveitamento agroindustrial de resíduos sólidos provenientes do melão minimamente processado. Ciências e Tecnologia de Alimentos, Campinas, v.28, n.3 p.733-737, 2008. 
MOTA, R.V. Caracterização física e química de geleia de amora-preta. Ciência e Tecnologia de Alimentos, v.26, n.3, p.539-543, 2006.

MUHAMMAD, A.; DURRANI, Y.; ZEB, A.; AYUB, M.; ULLAH, J. Development of diet jam from apple grown in swat (NWFP). Sarhad Journal of Agriculture, v.24, n.3, p.461-467, 2008.

NACHTIGALL, A. M.; SOUZA, E. L. de; MALGARIM, M.B.; ZAMBIAZI, R. C. Geleias light de amora-preta. Boletim do Centro de Pesquisa e Processamento de Alimentos, n. 2, p. 337-354, 2004.

NUNES, G.H.S.; MEDEIROS, A.C.; ARAUJO, E.L.; NOGUEIRA, C.H.F.; SOMBRA, K.D.S. Resistência de acessos de meloeiro à mosca-minadora Liriomyza spp. (Diptera: Agromyzidae). Revista Brasileira de Fruticultura, v.35, n.3, p.746-754,2013.

OLIVEIRA, R.G.; GODOY, H.T.; PRADO, M.A. Otimização de metodologia colorimétrica para a determinação de ácido ascórbico em geleias de frutas. Ciência e Tecnologia Alimentar, v.30, n.1, p.244-249, 2010.

OLIVEIRA, E. N. A.; ROCHA, A. P. T.; GOMES, J. P.; SANTOS, D. C. Processamento e caracterização físicoquímica de geleias diet de umbu-cajá (Spondias spp.). Bioscience Journal, v. 30, n. 4, p. 1007-1016, 2014.

ORDÓNEZ-SANTOS, L.E.; VÁSQUEZ-RIASCOS, A. Effect of processing and storage time on the vitamin $\mathrm{C}$ and lycopene contents of nectar of pink guava (Psidium guajava L.). Archivos Latinoamericanos de Nutricion, v. 60 , n. 3 , p. $280-284,2010$.

SAFDAR, M.N.; MUMTAZ, A.; HAMEED, T.; SIDDIQUI, N.; KHALIL, S.; AMJAD, M. Storage, studies of jam prepared from different mango varieties. Pakistan Journal of Nutrition, v.11, n.7, p.555-561, 2012.

SELVAMUTHUKUMARAN, M.; KHANUM, F.; BAWA, A.S. Development of sea buckthorn mixed mfruit jelly. International Journal of Food Science \& Technology, v.42, n.4, p.403-410, 2007

SILVA, C.M.R.; MANGABA, M.A.; FARINAZZIMACHADO, F.M.V.; SHIGEMATSU, E. Elaboração de geleias mistas, nas formulações tradicional, light e diet a partir da casca do maracujá amarelo (Passiflora edulis flavicarpa Degener). Revista Brasileira de Tecnologia Agroindustrial, v.6, n.2, p.770-780, 2012.

SILVA, P.T.; LOPES, M.L.M.; VALENTE-MESQUITA, V.L. Efeito de diferentes processamentos sobre o teor de ácido ascórbico em suco de laranja utilizado na elaboração de bolo, pudim e geleia. Ciência e Tecnologia Alimentar, v.26, n.3, p.678-682, 2006.

SOUSA, P.H.M.; MAIA, G.A.; AZEREDO, H.M.C.; RAMOS, A.M.; FIGUEIREDO, R.W. Sorage stability of a tropical fruit (cashew, apple, acerola, papaya, guava and passion fruit) mixed nectar added caffeine. International Journal of Food Science \& Technology, v.45, n.10, p.2162-2166, 2010.

SOUZA FILHO, M.S.; LIMA, J.R.; NASSU, R.T.; BORGES, M.F.; MOURA, C.F.H. Avaliação físico-química e sensorial de néctares de frutas nativas da região norte e nordeste do Brasil: estudo exploratório. Brazilian Journal of Food technology, Campinas, v.5, p.139-143, 2002.

TOMAZ, H.V.Q., AROUCHA, E.M.M., NUNES, G.H.S., BEZERRA NETO,F., TOMAZ, H.V.Q., QUEIROZ, R.F. Qualidade pós-colheita de diferentes híbridos de melão amarelo armazenados sob refrigeração. Revista Brasileira de Fruticultura, v.31, n.4, p. 987-994, 2009. 